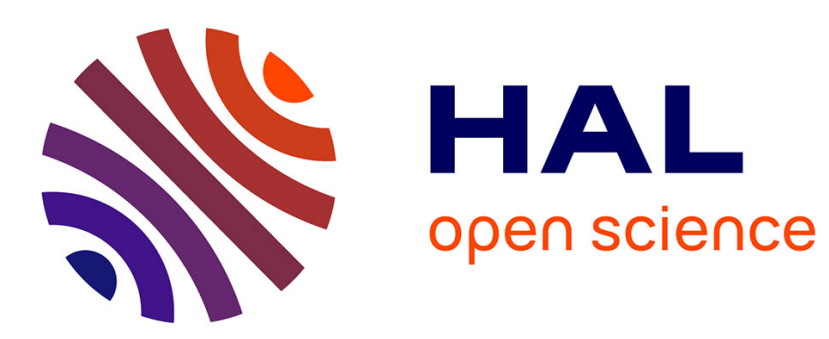

\title{
On the continuity of the eigenvalues of a sublaplacian
}

Amine Aribi, Sorin Dragomir, Ahmad El Soufi

\section{To cite this version:}

Amine Aribi, Sorin Dragomir, Ahmad El Soufi. On the continuity of the eigenvalues of a sublaplacian. Canadian Mathematical Bulletin, 2014, 57 (1), pp.12-24. 10.4153/CMB-2012-026-9 . hal-00923208

\section{HAL Id: hal-00923208 https://hal.science/hal-00923208}

Submitted on 2 Jan 2014

HAL is a multi-disciplinary open access archive for the deposit and dissemination of scientific research documents, whether they are published or not. The documents may come from teaching and research institutions in France or abroad, or from public or private research centers.
L'archive ouverte pluridisciplinaire HAL, est destinée au dépôt et à la diffusion de documents scientifiques de niveau recherche, publiés ou non, émanant des établissements d'enseignement et de recherche français ou étrangers, des laboratoires publics ou privés. 


\title{
On the continuity of the eigenvalues of a sublaplacian
}

\author{
Amine Aribi, Sorin Dragomir ${ }^{1}$, Ahmad El Soufi ${ }^{2}$
}

\begin{abstract}
AвstRact. We study the behavior of the eigenvalues of a sublaplacian $\Delta_{b}$ on a compact strictly pseudoconvex CR manifold $M$, as functions on the set $\mathcal{P}_{+}$of positively oriented contact forms on $M$ by endowing $\mathcal{P}_{+}$with a natural metric topology.
\end{abstract}

\section{INTRODUCTION}

Let $M$ be a compact strictly pseudoconvex CR manifold, of CR dimension $n$, without boundary. Let $\mathcal{P}$ be the set of all $C^{\infty}$ pseudohermitian structures on $M$. Every $\theta \in \mathcal{P}$ is a contact form on $M$ i.e. $\theta \wedge(d \theta)^{n}$ is a volume form. Let $\mathcal{P}_{ \pm}$be the sets of $\theta \in \mathcal{P}$ such that the Levi form $G_{\theta}$ is positive definite (respectively negative definite). For $\theta \in \mathcal{P}_{+}$let $\Delta_{b}$ be the sublaplacian

$$
\Delta_{b} u=-\operatorname{div}\left(\nabla^{H} u\right)
$$

of $(M, \theta)$ acting on smooth real valued functions $u \in C^{\infty}(M, \mathbb{R})$. As $\Delta_{b}$ is a subelliptic operator (of order 1/2) it has a discrete spectrum

$$
0=\lambda_{0}(\theta)<\lambda_{1}(\theta) \leq \lambda_{2}(\theta) \leq \cdots \uparrow+\infty
$$

(the eigenvalues of $\Delta_{b}$ are counted with their multiplicities). Each eigenvalue $\lambda_{v}(\theta), v=0,1,2, \cdots$, is thought of as a function of $\theta \in \mathcal{P}_{+}$. We shall deal mainly with the following problem: Is there a natural topology on $\mathcal{P}_{+}$such that each eigenvalue function $\lambda_{v}: \mathcal{P}_{+} \rightarrow \mathbb{R}$ is continuous? The analogous problem for the spectrum of the Laplace-Beltrami operator on a compact Riemannian manifold was solved by S. Bando \& H. Urakawa, [2], and our main result is imitative of their Theorem 2.2 (cf. op. cit., p. 155). We shall establish

Corollary 1. For every compact strictly pseudoconvex $C R$ manifold $M$ the space of positively oriented contact forms $\mathcal{P}_{+}$admits a natural complete

\footnotetext{
${ }^{1}$ Dipartimento di Matematica e Informatica, Università degli Studi della Basilicata, Viale dell'Ateneo Lucano 10, Campus Macchia Romana, 85100 Potenza, Italy, sorin.dragomir@unibas.it

${ }^{2}$ Laboratoire de Mathématiques et Physique Théorique, Université François Rabelais, Tours, France, amine.aribi@lmpt.univ-tours.fr, Ahmad.Elsoufi@lmpt. univ-tours.fr
} 
distance function $d: \mathcal{P}_{+} \times \mathcal{P}_{+} \rightarrow[0,+\infty)$ such that each eigenvalue function $\lambda_{k}: \mathcal{P}_{+} \rightarrow \mathbb{R}$ is continuous relative to the $d$-topology.

By a result of J.M. Lee, [8], for every $\theta \in \mathcal{P}_{+}$there is a Lorentzian metric $F_{\theta} \in \operatorname{Lor}(C(M)$ ) (the Fefferman metric) on the total space $C(M)$ of the canonical circle bundle $S^{1} \rightarrow C(M) \stackrel{\pi}{\rightarrow} M$. Also if $\square$ is the LaplaceBeltrami operator of $F_{\theta}$ (the wave operator) then $\sigma\left(\Delta_{b}\right) \subset \sigma(\square)$. Therefore the eigenvalues $\lambda_{k}$ may be thought of as functions $\lambda_{k}^{\uparrow}: C \rightarrow \mathbb{R}$ on the set $C=\left\{F_{\theta} \in \operatorname{Lor}(C(M)): \theta \in \mathcal{P}_{+}\right\}$of all Fefferman metrics on $C(M)$. On the other hand $\operatorname{Lor}(C(M))$ may be endowed with the distance function $d_{g}^{\infty}$ considered by P. Mounoud, [10] (associated to a fixed Riemannian metric $g$ on $C(M))$ and hence $\left(C, d_{g}^{\infty}\right)$ is itself a metric space. It is then a natural question whether $\lambda_{k}^{\uparrow}$ are continuous functions relative to the $d_{g}^{\infty}$-topology.

The paper is organized as follows. In $\S 2$ we recall the needed material on $\mathrm{CR}$ and pseudohermitian geometry. The distance function $d$ (in Corollary 1 ) is built in $\S 3$. In $\S 4$ we establish a Max-Mini principle (cf. Proposition 2) for the eigenvalues of a sublaplacian. Then Corollary 1 follows from Theorem 1 in $\S 5$. In $\S 6$ we prove the continuity of the eigenvalues with respect to the Fefferman metric (cf. Corollary 2) though only as functions on $C_{+}=\left\{e^{u \circ \pi} F_{\theta_{0}}: u \in C^{\infty}(M, \mathbb{R}), u>0\right\}$.

\section{REVIEW OF CR AND PSEUDOHERMITIAN GEOMETRY}

Let $\left(M, T_{1,0}(M)\right)$ be a CR manifold, of CR dimension $n$, where $T_{1,0}(M) \subset$ $T(M) \otimes \mathbb{C}$ is its CR structure. Cf. e.g. [5], p. 3-4. The Levi distribution is $H(M)=\operatorname{Re}\left\{T_{1,0}(M) \oplus \overline{T_{1,0}(M)}\right\}$. The Levi distribution carries the complex structure $J: H(M) \rightarrow H(M)$ given by $J(Z-\bar{Z})=i(Z-\bar{Z})$ for any $Z \in$ $T_{1,0}(M)$ (here $i=\sqrt{-1}$ ). A pseudohermitian structure is a globally defined nowhere zero section $\theta \in C^{\infty}\left(H(M)^{\perp}\right)$ in the conormal bundle $H(M)^{\perp} \subset$ $T^{*}(M)$. Pseudohermitian structures do exist by the mere assumption that $M$ be orientable. Let $\mathcal{P}$ be the set of all pseudohermitian structures on $M$. As $H(M)^{\perp} \rightarrow M$ is a real line bundle for any $\theta, \theta_{0} \in \mathcal{P}$ there is a $C^{\infty}$ function $\lambda: M \rightarrow \mathbb{R} \backslash\{0\}$ such that $\theta=\lambda \theta_{0}$. Given $\theta \in \mathcal{P}$ the Levi form is $G_{\theta}(X, Y)=(d \theta)(X, J Y)$ for every $X, Y \in \mathfrak{X}(M)$. Then $G_{\lambda \theta_{0}}=\lambda G_{\theta_{0}}$. The CR manifold $M$ is strictly pseudoconvex if $G_{\theta}$ is positive definite (write $G_{\theta}>0$ ) for some $\theta \in \mathcal{P}$. If $M$ is strictly pseudoconvex then each $\theta \in \mathcal{P}$ is a contact form i.e. $\Psi_{\theta}=\theta \wedge(d \theta)^{n}$ is a volume form on $M$. Clearly, if $G_{\theta}$ is positive definite then $G_{-\theta}$ is negative definite. Hence $\mathcal{P}$ admits a natural orientation $\mathcal{P}_{+}\left(G_{\theta}>0\right.$ for each $\left.\theta \in \mathcal{P}_{+}\right)$. Let $M$ be a strictly pseudoconvex $\mathrm{CR}$ manifold and $\theta \in \mathcal{P}_{+}$. The Reeb vector field is the globally defined, nowhere zero, tangent vector field $T \in \mathfrak{X}(M)$, transverse to $H(M)$, determined by $\theta(T)=1$ and $(d \theta)(T, X)=0$ for any $X \in \mathfrak{X}(M)$ (cf. Proposition 1.2 in [5], p. 8). The 
Webster metric is the Riemannian metric $g_{\theta}$ on $M$ given by

$$
g_{\theta}(X, Y)=G_{\theta}(X, Y), \quad g_{\theta}(X, T)=0, \quad g_{\theta}(T, T)=1,
$$

for every $X, Y \in H(M)$. Let $S^{1} \rightarrow C(M) \stackrel{\pi}{\longrightarrow} M$ be the canonical circle bundle (cf. Definition 2.9 in [5], p. 119). For every $\theta \in \mathcal{P}_{+}$there is a Lorentzian metric $F_{\theta}$ on $C(M)$ (the Fefferman metric, cf. Definition 2.15 in [5], p. 128) such that the set $C=\left\{F_{\theta}: \theta \in \mathcal{P}_{+}\right\}$of all Fefferman metrics is given by $C=\left\{e^{u \circ \pi} F_{\theta}: u \in C^{\infty}(M, \mathbb{R})\right\}$ for each fixed contact form $\theta \in \mathcal{P}_{+}$ (by a result of J.M. Lee, [8], or Theorem 2.3 in [5], p. 128). $C$ is also referred to as the restricted conformal class of $F_{\theta}$ and it is a CR invariant.

If $u \in C^{\infty}(M, \mathbb{R})$ then the horizontal gradient $\nabla^{H} u \in C^{\infty}(H(M))$ is given by $\nabla^{H} u=\Pi_{H} \nabla u$. Here $\Pi_{H}: T(M) \rightarrow H(M)$ is the projection relative to the decomposition $T(M)=H(M) \oplus \mathbb{R} T$ and $\nabla u$ is the gradient of $u$ with respect to the Webster metric i.e. $g_{\theta}(\nabla u, X)=X(u)$ for any $X \in \mathfrak{X}(M)$. The divergence operator div : $\mathfrak{X}(M) \rightarrow C^{\infty}(M, \mathbb{R})$ is meant with respect to the volume form $\Psi_{\theta}$ i.e. $\mathcal{L}_{X} \Psi_{\theta}=\operatorname{div}(X) \Psi_{\theta}$ for any $X \in \mathfrak{X}(M)$. The sublaplacian $\Delta_{b}$ of $(M, \theta)$ is then the formally self-adjoint, second order, degenerate elliptic (in the sense of J.M. Bony, [4]) operator given by $\Delta_{b} u=-\operatorname{div}\left(\nabla^{H} u\right)$ for any $u \in C^{\infty}(M, \mathbb{R})$. A systematic application of functional analysis methods to the study of sublaplacians (on domains in strictly pseudoconvex CR manifolds) was started in [3]. By a result following essentially from work in [9] (cf. also [12]) if $M$ is compact then $\Delta_{b}$ has a discrete spectrum $\sigma\left(\Delta_{b}\right)=\left\{\lambda_{v}: v \geq 0\right\}$ such that $\lambda_{0}=0$ and $\lambda_{v} \uparrow+\infty$ as $v \rightarrow \infty$.

\section{A TOPOLOGY ON THE SPACE OF ORIENTED CONTACT FORMS}

Let $\left\{U_{\lambda}\right\}_{\lambda \in \Lambda}$ be a finite open covering of $M$ such that the closure of each $U_{\lambda}$ is contained in a larger open set $V_{\lambda}$ which is both the domain of a local frame $\left\{X_{a}: 1 \leq a \leq 2 n\right\} \subset C^{\infty}\left(V_{\lambda}, H(M)\right)$ with $X_{\alpha+n}=J X_{\alpha}$ for any $1 \leq \alpha \leq$ $n$, and a coordinate neighborhood with the local coordinates $\left(x^{1}, \cdots, x^{2 n+1}\right)$. For each point $x \in M$ let $P_{x}$ (respectively $S_{x}$ ) be the set of all symmetric positive definite (respectively merely symmetric) bilinear forms on $T_{x}(M)$. Let us consider the anti-reflexive partial order relation on $S_{x}$ defined by

$$
\varphi<\psi \Longleftrightarrow \psi-\varphi \in P_{x}, \quad \varphi, \psi \in S_{x} .
$$

Next let $\rho_{x}^{\prime \prime}: P_{x} \times P_{x} \rightarrow[0,+\infty)$ be the distance function given by

$$
\rho_{x}^{\prime \prime}(\varphi, \psi)=\inf \{\delta>0: \exp (-\delta) \varphi<\psi<\exp (\delta) \varphi\}
$$

for any $\varphi, \psi \in P_{x}$. Then $\left(P_{x}, \rho_{x}^{\prime \prime}\right)$ is a complete metric space (by (iii) of Lemma 1.1 in [2], p. 158).

Let $\mathcal{M}$ be the set of all Riemannian metrics on $M$, so that $g_{\theta} \in \mathcal{M}$ for every $\theta \in \mathcal{P}_{+}$. Following [2] one may endow $\mathcal{M}$ with a complete distance 
function $\rho$. Indeed as $M$ is compact one may set

$$
\rho^{\prime \prime}\left(g_{1}, g_{2}\right)=\sup _{x \in M} \rho_{x}^{\prime \prime}\left(g_{1, x}, g_{2, x}\right), \quad g_{1}, g_{2} \in \mathcal{M} .
$$

Also let $S(M)$ be the space of all $C^{\infty}$ symmetric $(0,2)$-tensor fields on $M$, organized as a Fréchet space by the family of seminorms $\left\{|\cdot|_{k}: k \in \mathbb{N} \cup\{0\}\right\}$ where

$$
|g|_{k}=\sum_{\lambda \in \Lambda}|g|_{\lambda, k}, \quad|g|_{\lambda, k}=\sup _{x \in \bar{U}_{\lambda}} \sum_{|\alpha| \leq k}\left|D^{\alpha} g_{i j}(x)\right|,
$$

where

$$
D^{\alpha}=\partial^{|\alpha|} / \partial\left(x^{1}\right)^{\alpha_{1}} \cdots \partial\left(x^{2 n+1}\right)^{\alpha_{2 n+1}}, \quad g_{i j}=g\left(\partial / \partial x^{i}, \partial / \partial x^{j}\right) \in C^{\infty}\left(V_{\lambda}, \mathbb{R}\right),
$$

for any $g \in S(M)$. The topology of $S(M)$ as a locally convex space is compatible to the distance function

$$
\rho^{\prime}\left(g_{1}, g_{2}\right)=\sum_{k=0}^{\infty} \frac{1}{2^{k}} \frac{\left|g_{1}-g_{2}\right|_{k}}{1+\left|g_{1}-g_{2}\right|_{k}}, \quad g_{1}, g_{2} \in S(M) .
$$

In particular $\left(S(M), \rho^{\prime}\right)$ is a complete metric space. If

$$
\rho\left(g_{1}, g_{2}\right)=\rho^{\prime}\left(g_{1}, g_{2}\right)+\rho^{\prime \prime}\left(g_{1}, g_{2}\right)
$$

then $(\mathcal{M}, \rho)$ is a complete metric space (cf. Proposition 2 in [2], p. 158). Each metric $g \in \mathcal{M}$ determines a Laplace-Beltrami operator $\Delta_{g}$ hence the eigenvalues of $\Delta_{g}$ may be though of as functions of $g$ and as such the eigenvalues are (by Theorem 2.2 in [2], p. 161) continuous functions on $(\mathcal{M}, \rho)$. To deal with the similar problem for the spectrum of a sublaplacian, we start by observing that the natural counterpart of $\mathcal{M}$ in the category of strictly pseudoconvex CR manifolds is the set $\mathcal{M}_{H}$ of all sub-Riemannian metrics on $(M, H(M))$. Nevertheless only a particular sort of sub-Riemannian metric gives rise to a sublaplacian i.e. $\Delta_{b}$ is associated to $G_{\theta} \in \mathcal{M}_{H}$ for some positively oriented contact form $\theta \in \mathcal{P}_{+}$. Of course $\mathcal{P}_{+} \subset \Omega^{1}(M)$ and one may endow $\Omega^{1}(M)$ with the $C^{\infty}$ topology. One may then attempt to repeat the arguments in [2] (by replacing $S(M)$ with $\Omega^{1}(M)$ ). The situation at hand is however much simpler since, once a contact form $\theta_{0} \in \mathcal{P}_{+}$is fixed, all others are parametrized by $C^{\infty}(M, \mathbb{R})$ i.e. for any $\theta \in \mathcal{P}_{+}$there is a unique $u \in C^{\infty}(M, \mathbb{R})$ such that $\theta=e^{u} \theta_{0}$. We may then use the canonical Fréchet space structure (and corresponding complete distance function) of $C^{\infty}(M, \mathbb{R})$. Precisely, for every $u \in C^{\infty}(M, \mathbb{R}), \lambda \in \Lambda$ and $k \in \mathbb{N} \cup\{0\}$ we set

$$
\begin{gathered}
p_{\lambda, k}(u)=\sup _{x \in \bar{U}_{k}} \sum_{|\alpha| \leq k}\left|D^{\alpha} u(x)\right|, \\
p_{k}(u)=\sum_{\lambda \in \Lambda} p_{\lambda, k}(u), \quad|u|_{C^{\infty}}=\sum_{k=0}^{\infty} \frac{1}{2^{k}} \frac{p_{k}(u)}{1+p_{k}(u)} .
\end{gathered}
$$


If $\theta_{0} \in \mathcal{P}_{+}$is a fixed contact form then we set

$$
d^{\prime}\left(\theta_{1}, \theta_{2}\right)=\left|u_{1}-u_{2}\right|_{C^{\infty}}, \quad \theta_{1}, \theta_{2} \in \mathcal{P}_{+},
$$

where $u_{i} \in C^{\infty}(M, \mathbb{R})$ are given by $\theta_{i}=e^{u_{i}} \theta_{0}$ for any $i \in\{1,2\}$. The definition of $d^{\prime}$ doesn't depend upon the choice of $\theta_{0} \in \mathcal{P}_{+}$.

Lemma 1. $\left(\mathcal{P}_{+}, d^{\prime}\right)$ is a complete metric space.

Proof. Let $\left\{\theta_{v}\right\}_{v \geq 1}$ be a Cauchy sequence in $\left(\mathcal{P}_{+}, d^{\prime}\right)$. If $u_{v} \in C^{\infty}(M, \mathbb{R})$ is the function determined by $\theta_{v}=e^{u_{v}} \theta_{0}$ then (by the very definition of $d^{\prime}$ ) $\left\{u_{v}\right\}_{v \geq 1}$ is a Cauchy sequence in $C^{\infty}(M, \mathbb{R})$. Here $C^{\infty}(M, \mathbb{R})$ is organized as a Fréchet space by the (countable, separating) family of seminorms $\left\{p_{k}: k \in\right.$ $\mathbb{N} \cup\{0\}\}$. Hence there is $u \in C^{\infty}(M, \mathbb{R})$ such that $\left|u_{v}-u\right|_{C^{\infty}} \rightarrow 0$ as $v \rightarrow \infty$. Finally if $\theta=e^{u} \theta_{0} \in \mathcal{P}_{+}$then $d^{\prime}\left(\theta_{v}, \theta\right) \rightarrow 0$ as $v \rightarrow \infty$. Q.e.d.

Let $S(H) \subset H(M)^{*} \otimes H(M)^{*}$ be the subbundle of all bilinear symmetric forms on $H(M)$. For every $G \in C^{\infty}(S(H)), k \in \mathbb{Z}, k \geq 0$, and $\lambda \in \Lambda$ we set

$$
\begin{gathered}
|G|_{\lambda, k}=\sup _{x \in \bar{U}_{\lambda}} \sum_{|\alpha| \leq k} \sum_{a, b=1}^{2 n}\left|D^{\alpha} G_{a b}(x)\right|, \\
|G|_{k}=\sum_{\lambda \in \Lambda}|G|_{\lambda, k}, \quad|G|_{C^{\infty}}=\sum_{k=0}^{\infty} \frac{1}{2^{k}} \frac{|G|_{k}}{1+|G|_{k}},
\end{gathered}
$$

where $G_{a b}=G\left(X_{a}, X_{b}\right) \in C^{\infty}\left(V_{\lambda}, \mathbb{R}\right)$. Moreover we set

$$
\rho_{H}^{\prime}\left(G_{1}, G_{2}\right)=\left|G_{1}-G_{2}\right|_{C^{\infty}}, \quad G_{1}, G_{2} \in C^{\infty}(S(H)) .
$$

Lemma 2. $\left\{|\cdot|_{k}: k \in \mathbb{N} \cup\{0\}\right\}$ is a countable separating family of seminorms organizing $\mathfrak{X}=C^{\infty}(S(H))$ as a Fréchet space. In particular $\left(\mathfrak{X}, \rho_{H}^{\prime}\right)$ is a complete metric space.

Proof. For each $k \in \mathbb{N} \cup\{0\}$ and $N \in \mathbb{N}$ we set

$$
V(k, N)=\left\{G \in \mathfrak{X}:|G|_{k}<1 / N\right\} .
$$

Let $\mathcal{B}$ be the collection of all finite intersections of sets (3). Then $\mathcal{B}$ is (cf. e.g. Theorem 1.37 in [11], p. 27) a convex balanced local base for a topology $\tau$ on $\mathfrak{X}$ which makes $\mathfrak{X}$ into a locally convex space such that every seminorm $|\cdot|_{k}$ is continuous and a set $E \subset \mathfrak{X}$ is bounded if and only if every $|\cdot|_{k}$ is bounded on $E$. $\tau$ is compatible with the distance function $\rho_{H}^{\prime}$. Let $\left\{G_{m}\right\}_{m \geq 1} \subset \mathfrak{X}$ be a Cauchy sequence relative to $\rho_{H}^{\prime}$. Thus for every fixed $k \in \mathbb{N} \cup\{0\}$ and $N \in \mathbb{N}$ one has $G_{m}-G_{p} \in V(k, N)$ for $m, p$ sufficiently large. Consequently

$$
\begin{gathered}
\left|D^{\alpha}\left(G_{m}\right)_{a b}(x)-D^{\alpha}\left(G_{p}\right)_{a b}(x)\right|<1 / N, \\
x \in \bar{U}_{\lambda}, \quad \lambda \in \Lambda, \quad|\alpha| \leq k, \quad 1 \leq a, b \leq 2 n .
\end{gathered}
$$


It follows that each sequence $\left\{D^{\alpha}\left(G_{m}\right)_{a b}\right\}_{m \geq 1}$ converges uniformly on $\bar{U}_{\lambda}$ to a function $G_{a b}^{\alpha}$. In particular for $\alpha=\mathbf{0}$ one has $\left(G_{m}\right)_{a b}(x) \rightarrow G_{a b}^{\mathbf{0}}(x)$ as $m \rightarrow \infty$, uniformly in $x \in \bar{U}_{\lambda}$. If $\lambda, \lambda^{\prime} \in \Lambda$ are such that $U_{\lambda} \cap U_{\lambda^{\prime}} \neq \emptyset$ and

$$
X_{b}^{\prime}=A_{b}^{a} X_{a}, \quad A \equiv\left[A_{b}^{a}\right]: U_{\lambda} \cap U_{\lambda^{\prime}} \rightarrow \operatorname{GL}(2 n, \mathbb{R}),
$$

is a local transformation of the frame in $H(M)$ then

$$
\left(G_{m}\right)_{a b}^{\prime}=A_{a}^{c} A_{b}^{d}\left(G_{m}\right)_{c d} \text { on } U_{\lambda} \cap U_{\lambda^{\prime}}
$$

so that (for $m \rightarrow \infty$ ) $G_{a b}^{\prime \mathbf{0}}=A_{a}^{c} A_{b}^{d} G_{c d}^{\mathbf{0}}$ on $U_{\lambda} \cap U_{\lambda^{\prime}}$. Thus $G_{a b}^{\mathbf{0}} \in C^{\infty}\left(U_{\lambda}\right)$ glue up to a (globally defined) bilinear symmetric form $G^{\mathbf{0}}$ on $H(M)$ and $G_{m} \rightarrow G^{\mathbf{0}}$ in $\mathfrak{X}$ as $m \rightarrow \infty$. Q.e.d.

For each point $x \in M$ let $P(H)_{x}$ be the set of all symmetric positive definite bilinear forms on $H(M)_{x}$. We endow $S(H)_{x}$ with the anti-reflexive partial order relation

$$
\varphi<\psi \Longleftrightarrow \psi-\varphi \in P(H)_{x}, \quad \varphi, \psi \in S(H)_{x} .
$$

Next let $\rho_{x}^{\prime \prime}: P(H)_{x} \times P(H)_{x} \rightarrow[0,+\infty)$ be given by

$$
\rho_{x}^{\prime \prime}(\varphi, \psi)=\inf \{\delta>0: \exp (-\delta) \varphi<\psi<\exp (\delta) \varphi\}
$$

for any $\varphi, \psi \in P(H)_{x}$.

Lemma 3. $\rho_{x}^{\prime \prime}$ is a distance function on $P(H)_{x}$.

Proof. As $e^{-\delta} \varphi<\psi<e^{\delta} \varphi$ is equivalent to $e^{-\delta} \psi<\varphi<e^{\delta} \psi$, it follows that $\rho_{x}^{\prime \prime}$ is symmetric. To prove the triangle inequality we assume that $\rho_{x}^{\prime \prime}(\varphi, \psi)>$ $\rho_{x}^{\prime \prime}(\varphi, \chi)+\rho^{\prime \prime}(\chi, \psi)$ for some $\varphi, \psi, \chi \in P(H)_{x}$. Then

$$
\rho_{x}^{\prime \prime}(\varphi, \psi)-\rho_{x}^{\prime \prime}(\varphi, \chi)>\inf \{\delta>0: \exp (-\delta) \chi<\psi<\exp (\delta) \chi\}
$$

hence there is $\delta_{2}>0$ such that $e^{-\delta_{2}} \chi<\psi<e^{\delta_{2}} \chi$ and $\rho_{x}^{\prime \prime}(\varphi, \psi)-\rho_{x}^{\prime \prime}(\varphi, \chi)>\delta_{2}$. Similarly

$$
\rho_{x}^{\prime \prime}(\varphi, \psi)-\delta_{2}>\inf \{\delta>0: \exp (-\delta) \varphi<\chi<\exp (\delta) \varphi\}
$$

yields the existence of a number $\delta_{1}>0$ such that $e^{-\delta_{1}} \varphi<\chi<e^{\delta_{1}} \varphi$ and $\rho_{x}^{\prime \prime}(\varphi, \psi)-\delta_{2}>\delta_{1}$. Let us set $\delta \equiv \delta_{1}+\delta_{2}$. The inequalities written so far show that $e^{-\delta} \varphi<\psi<e^{\delta} \varphi$ and $\rho_{x}^{\prime \prime}(\varphi, \psi)>\delta$, a contradiction. Finally, let us assume that $\rho_{x}^{\prime \prime}(\varphi, \psi)=0$ so that for any $k \in \mathbb{N}$

$$
\inf \{\delta>0: \exp (-\delta) \varphi<\psi<\exp (\delta) \varphi\}<1 / k
$$

i.e. there is $\delta_{k}>0$ such that $e^{-\delta_{k}} \varphi<\psi<e^{\delta_{k}} \varphi$ and $\delta_{k}<1 / k$. Thus $\lim _{k \rightarrow \infty} \delta_{k}=0$ and $\psi-e^{-\delta_{k}} \varphi \in P(H)_{x}$ shows (by passing to the limit with $k \rightarrow \infty$ in $\left.\psi(v, v)-e^{-\delta_{k}} \varphi(v, v)>0, v \in H(M)_{x} \backslash\{0\}\right)$ that $\varphi<\psi$. Similarly $e^{\delta_{k}} \varphi-\psi \in P(H)_{x}$ yields in the limit $\psi<\varphi$, and we may conclude that $\varphi=\psi$. Viceversa, if $\varphi \in P(H)_{x}$ then

$$
\left\{\delta>0:\left(1-e^{-\delta}\right) \varphi,\left(e^{\delta}-1\right) \varphi \in P(H)_{x}\right\}=(0,+\infty)
$$


hence $\rho_{x}^{\prime \prime}(\varphi, \varphi)=0$. Q.e.d.

Lemma 4. i) $\left(P(H)_{x}, \rho_{x}^{\prime \prime}\right)$ is a complete metric space.

ii) Let $\left\{\varphi_{j}\right\}_{j \in \mathbb{N}} \subset P(H)_{x}$ such that $\lim _{j \rightarrow \infty} \varphi_{j}=\varphi \in P(H)_{x}$ in the $\rho_{x}^{\prime \prime}$ topology. Then $\lim _{j \rightarrow \infty} \varphi_{j}(v, w)=\varphi(v, w)$ for any $v, w \in H(M)_{x}$.

Proof. i) Let $\left\{\varphi_{j}\right\}_{j \in \mathbb{N}} \subset P(H)_{x}$ be a Cauchy sequence in the $\rho_{x}^{\prime \prime}$-topology i.e. for any $\epsilon>0$ there is $j_{\epsilon} \in \mathbb{N}$ such that $\rho_{x}^{\prime \prime}\left(\varphi_{j+p}, \varphi_{j}\right)>\epsilon$ for any $j \geq j_{\epsilon}$ and any $p=1,2, \cdots$. Hence there is $\delta_{\epsilon}>0$ such that $e^{-\delta_{\epsilon}} \varphi_{j}<\varphi_{j+p}<e^{\delta_{\epsilon}} \varphi_{j}$ and $\delta_{\epsilon}<\epsilon$. Consequently

$$
\left|\log \varphi_{j+p}(v, v)-\log \varphi_{j}(v, v)\right|<\delta_{\epsilon}<\epsilon
$$

for any $v \in H(M)_{x} \backslash\{0\}$. Therefore if

$$
\xi_{j} \equiv\left(\log \varphi_{j}(v, v), \cdots, \log \varphi_{j}(v, v)\right) \in \mathbb{R}^{2 n}
$$

then $\left\{\xi_{j}\right\}_{j \in \mathbb{N}}$ is a Cauchy sequence in $\mathbb{R}^{2 n}$. Let then $\xi=\lim _{j \rightarrow \infty} \xi_{j}$ and let $\varphi: H(M)_{x} \times H(M)_{x} \rightarrow \mathbb{R}$ be the bilinear form given by $\varphi(v, v)=\exp \left(\xi^{a}\right)$ for any $v \in H(M)_{x} \backslash\{0\}$ followed by polarization. Here $\xi=\left(\xi^{1}, \cdots, \xi^{2 n}\right)$. Then $\varphi \in P(H)_{x}$ and $\lim _{j \rightarrow \infty} \varphi_{j}=\varphi$ in the $\rho_{x}^{\prime \prime}$-topology.

ii) If $\varphi_{j} \rightarrow \varphi$ as $j \rightarrow \infty$ then $\log \varphi_{j}(v, v) \rightarrow \log \varphi(v, v)$ as $j \rightarrow \infty$, for any $v \in H(M)_{x} \backslash\{0\}$. Then $\lim _{j \rightarrow \infty} \varphi_{j}(v, v)=\varphi(v, v)$ uniformly in $v$ and statement (ii) follows by polarization. Q.e.d.

As $M$ is compact we may set

$$
\begin{gathered}
\rho_{H}^{\prime \prime}\left(G_{1}, G_{2}\right)=\sup _{x \in M} \rho_{x}^{\prime \prime}\left(G_{1, x}, G_{2, x}\right), \\
\rho_{H}\left(G_{1}, G_{2}\right)=\rho_{H}^{\prime}\left(G_{1}, G_{2}\right)+\rho_{H}^{\prime \prime}\left(G_{1}, G_{2}\right), \quad G_{1}, G_{2} \in \mathcal{M}_{H} .
\end{gathered}
$$

Also let $d$ be the distance function on $\mathcal{P}_{+}$given by

$$
d\left(\theta_{1}, \theta_{2}\right)=d^{\prime}\left(\theta_{1}, \theta_{2}\right)+\rho_{H}^{\prime \prime}\left(G_{\theta_{1}}, G_{\theta_{2}}\right), \quad \theta_{1}, \theta_{2} \in \mathcal{P}_{+} .
$$

Proposition 1. i) $\left(\mathcal{M}_{H}, \rho_{H}\right)$ is a complete metric space.

ii) The map $\theta \in \mathcal{P}_{+} \mapsto G_{\theta} \in \mathcal{M}_{H}$ of $\left(\mathcal{P}_{+}, d\right)$ into $\left(\mathcal{M}_{H}, \rho_{H}\right)$ is continuous.

iii) $\left(\mathcal{P}_{+}, d\right)$ is a complete metric space.

Proof. i) Let $\left\{G_{j}\right\}_{j \geq 1}$ be a Cauchy sequence in $\left(\mathcal{M}_{H}, \rho_{H}\right)$. Then $\left\{G_{j}\right\}_{j \geq 1}$ is a Cauchy sequence in both $\left(\mathfrak{X}, \rho_{H}^{\prime}\right)$ and $\left(\mathcal{M}_{H}, \rho_{H}^{\prime \prime}\right)$. Yet $\left(\mathfrak{X}, \rho_{H}^{\prime}\right)$ is complete (by Lemma 2). Thus $\rho_{H}^{\prime}\left(G_{j}, G\right) \rightarrow 0$ as $j \rightarrow \infty$ for some $G \in \mathfrak{X}$. In particular

$$
\lim _{j \rightarrow \infty} G_{j, x}(v, w)=G_{x}(v, w)
$$

for every $x \in M$ and $v, w \in H(M)_{x}$. On the other hand, as $\left\{G_{j}\right\}_{j \geq 1}$ is Cauchy in $\left(\mathcal{M}_{H}, \rho_{H}^{\prime \prime}\right)$, for every $\epsilon>0$ there is $N_{\epsilon} \geq 1$ such that

$$
\rho_{x}^{\prime \prime}\left(G_{i, x}, G_{j, x}\right) \leq \rho_{H}^{\prime \prime}\left(G_{i}, G_{j}\right)<\epsilon
$$


for every $i, j \geq N_{\epsilon}$ and $x \in M$. Thus $\left\{G_{j, x}\right\}_{j \geq 1}$ is Cauchy in the complete (by Lemma 4) metric space $\left(P(H)_{x}, \rho_{x}^{\prime \prime}\right)$ so that $\rho_{x}^{\prime \prime}\left(G_{j, x}, \varphi\right) \rightarrow 0$ as $j \rightarrow \infty$ for some $\varphi \in P(H)_{x}$. Then (by (iii) in Lemma 4) $\lim _{j \rightarrow \infty} G_{j, x}(v, w)=\varphi(v, w)$ for every $v, w \in H(M)_{x}$ hence $G_{x}=\varphi$ yielding $G \in \mathcal{M}_{H}$.

ii) Let $\left\{\theta_{v}\right\}_{v \geq 1} \subset \mathcal{P}_{+}$such that $d\left(\theta_{v}, \theta\right) \rightarrow 0$ for $v \rightarrow \infty$ for some $\theta \in \mathcal{P}_{+}$. If $\theta_{v}=e^{u_{v}} \theta_{0}$ and $\theta=e^{u} \theta_{0}$ then $\left|u_{v}-u\right|_{C^{\infty}} \rightarrow 0$ as $v \rightarrow \infty$. Then $G_{\theta_{v}}=e^{u_{v}} G_{\theta_{0}}$ and $G_{\theta}=e^{u} G_{\theta_{0}}$. Since $D^{\alpha} u_{v} \rightarrow D^{\alpha} u$ as $v \rightarrow \infty$, uniformly on $\bar{U}_{\lambda}$, for any $\lambda \in \Lambda,|\alpha| \leq k$ and $k \in \mathbb{N} \cup\{0\}$, it follows that $D^{\alpha}\left(G_{\theta_{v}}\right)_{a b} \rightarrow D^{\alpha}\left(G_{\theta}\right)_{a b}$ as $v \rightarrow \infty$ uniformly on $\bar{U}_{\lambda}$ for any $1 \leq a, b \leq 2 n$. Hence $G_{\theta_{v}} \rightarrow G_{\theta}$ in $\mathfrak{X}$ so that (by the very definition of $d$ and $\left.\rho_{H}\right) \rho_{H}\left(G_{\theta_{v}}, G_{\theta}\right) \rightarrow 0$. Q.e.d.

iii) If $\left\{\theta_{v}\right\}_{v \geq 1}$ is a Cauchy sequence in $\left(\mathcal{P}_{+}, d\right)$ then $\left\{u_{v}\right\}_{v \geq 1}$ is Cauchy in $\left(\mathcal{P}_{+}, d^{\prime}\right)$ as well. Yet (by Lemma 1$)\left(\mathcal{P}_{+}, d^{\prime}\right)$ is complete hence $d^{\prime}\left(\theta_{v}, \theta\right) \rightarrow$ 0 for some $\theta \in \mathcal{P}_{+}$. Then, as a byproduct of the proof of statement (ii), one has $G_{\theta_{v}} \rightarrow G_{\theta}$ in $\mathfrak{X}$. Finally, the verbatim repetition of the arguments in the proof of statement (i) yields $\rho_{H}^{\prime \prime}\left(G_{\theta_{v}}, G_{\theta}\right) \rightarrow 0$ so that $d\left(\theta_{v}, \theta\right) \rightarrow 0$. Q.e.d.

\section{A MAX-MINI PRINCIPLE}

For each $k \in \mathbb{N} \cup\{0\}$ we consider a $(k+1)$-dimensional real subspace $L_{k+1} \subset C^{\infty}(M, \mathbb{R})$ and set

$$
\Lambda_{\theta}\left(L_{k+1}\right)=\sup \left\{\frac{\left\|\nabla^{H} f\right\|_{L^{2}}^{2}}{\|f\|_{L^{2}}^{2}}: f \in L_{k+1} \backslash\{0\}\right\} .
$$

Here

$$
\|f\|_{L^{2}}=\left(\int_{M} f^{2} \Psi_{\theta}\right)^{\frac{1}{2}}, \quad\|X\|_{L^{2}}=\left(\int_{M} g_{\theta}(X, X) \Psi_{\theta}\right)^{\frac{1}{2}},
$$

for any $f \in C^{\infty}(M, \mathbb{R})$ and any $X \in \mathfrak{X}(M)$. Let $\left\{u_{v}\right\}_{v \geq 0} \subset C^{\infty}(M, \mathbb{R})$ be a complete orthonormal system relative to the $L^{2}$ inner product $(f, g)_{L^{2}}=$ $\int_{M} f g \Psi_{\theta}$ such that $u_{v} \in \operatorname{Eigen}\left(\Delta_{b} ; \lambda_{v}(\theta)\right)$ for every $v \geq 0$. If $f \in C^{\infty}(M, \mathbb{R})$ then $f=\sum_{v=0}^{\infty} a_{v}(f) u_{v}\left(L^{2}\right.$ convergence) for some $a_{v}(f) \in \mathbb{R}$. Let $L_{k+1}^{0}$ be the subspace of $C^{\infty}(M, \mathbb{R})$ spanned by $\left\{u_{v}: 0 \leq v \leq k\right\}$. Let $\left(\nabla^{H}\right)^{*}$ be the formal adjoint of $\nabla^{H}$ i.e.

$$
\left(\nabla^{H} f, X\right)_{L^{2}}=\left(f,\left(\nabla^{H}\right)^{*} X\right)_{L^{2}}
$$

for any $f \in C^{\infty}(M, \mathbb{R})$ and $X \in C^{\infty}(H(M))$. Mere integration by parts shows that

$$
\left(\nabla^{H}\right)^{*} X=-\operatorname{div}(X), \quad X \in C^{\infty}(H(M)),
$$

implying (by (1)) the useful identity

$$
\left\|\nabla^{H} f\right\|_{L^{2}}^{2}=\left(f, \Delta_{b} f\right)_{L^{2}}, \quad f \in C^{\infty}(M, \mathbb{R}) .
$$


Let $f \in L_{k+1}^{0} \backslash\{0\}$ so that $f=\sum_{v=0}^{k} a_{v} u_{v}$ for some $a_{v} \in \mathbb{R}$. Then (by (6))

$$
\left\|\nabla^{H} f\right\|_{L^{2}}^{2}=\sum_{v=0}^{k} a_{v}^{2} \lambda_{v}(\theta) \leq \lambda_{k}(\theta) \sum_{v=0}^{k} a_{v}^{2}=\lambda_{k}(\theta)\|f\|_{L^{2}}^{2}
$$

hence

$$
\Lambda_{\theta}\left(L_{k+1}^{0}\right) \leq \lambda_{k}(\theta) .
$$

Our purpose in this section is to establish

Proposition 2. Let $M$ be a compact strictly pseudoconvex CR manifold and $\theta \in \mathcal{P}_{+}$a positively oriented contact form. Then

$$
\lambda_{k}(\theta)=\inf _{L_{k+1}} \Lambda_{\theta}\left(L_{k+1}\right)
$$

where the g.l.b. is taken over all subspaces $L_{k+1} \subset C^{\infty}(M, \mathbb{R})$ with $\operatorname{dim}_{\mathbb{R}} L_{k+1}=$ $k+1$.

So far (by (7)) $\lambda_{k}(\theta) \geq \Lambda_{\theta}\left(L_{k+1}^{0}\right) \geq \inf _{L_{k+1}} \Lambda_{\theta}\left(L_{k+1}\right)$. The proof of Proposition 2 is by contradiction. We assume that $\lambda_{k}(\theta)>\inf _{L_{k+1}} \Lambda_{\theta}\left(L_{k+1}\right)$ i.e. there is a $(k+1)$-dimensional subspace $L_{k+1} \subset C^{\infty}(M, \mathbb{R})$ such that $\Lambda_{\theta}\left(L_{k+1}\right)<$ $\lambda_{k}(\theta)$. Then $\Lambda_{\theta}\left(L_{k+1}\right)$ is finite and

$$
\|f\|_{L^{2}}^{2} \Lambda_{\theta}\left(L_{k+1}\right) \geq\left\|\nabla^{H} f\right\|_{L^{2}}^{2}, \quad f \in L_{k+1} .
$$

Then (by (6))

so that

$$
\sum_{v=0}^{\infty} a_{v}(f)^{2} \Lambda_{\theta}\left(L_{k+1}\right) \geq \sum_{v=0}^{\infty} \lambda_{v}(\theta) a_{v}(f)^{2}
$$

$$
\begin{aligned}
& \sum_{\Lambda_{\theta}\left(L_{k+1}\right) \geq \Lambda_{\nu}(\theta)} a_{v}(f)^{2}\left[\Lambda_{\theta}\left(L_{k+1}\right)-\lambda_{\nu}(\theta)\right] \geq \\
\geq & \sum_{\Lambda_{\theta}\left(L_{k+1}\right)<\lambda_{\nu}(\theta)} a_{\nu}(f)^{2}\left[\lambda_{\nu}(\theta)-\Lambda_{\theta}\left(L_{k+1}\right)\right] .
\end{aligned}
$$

Let $\Phi: L_{k+1} \rightarrow C^{\infty}(M, \mathbb{R})$ be the linear map given by

$$
\Phi(f)=\sum_{v=0}^{m} a_{v}(f) u_{v}, \quad f \in L_{k+1},
$$

where $m=\max \left\{v \geq 0: \lambda_{v}(\theta) \leq \Lambda_{\theta}\left(L_{k+1}\right)\right\}$. Note that $0 \leq m \leq k-1$ (by the contradiction assumption). We claim that

$$
\operatorname{Ker}(\Phi) \neq(0) \text {. }
$$

Of course (10) is only true within the contradiction loop. The statement follows from $\operatorname{dim}_{\mathbb{R}} \Phi\left(L_{k+1}\right) \leq m+1 \leq k<k+1$ (hence $\Phi$ cannot be injective). Let (by (10)) $f_{0} \in L_{k+1}$ such that $\Phi\left(f_{0}\right)=0$ and $f_{0} \neq 0$. Then $a_{v}\left(f_{0}\right)=0$ for any $0 \leq v \leq m$ i.e. whenever $\Lambda_{\theta}\left(L_{k+1}\right) \geq \lambda_{v}(\theta)$. Applying 
(9) to $f=f_{0}$ yields $a_{v}\left(f_{0}\right)=0$ whenever $\Lambda_{\theta}\left(L_{k+1}\right)<\lambda_{v}(\theta)$. Thus $f_{0}=0$, a contradiction.

\section{Continuity of eigenvalues}

The scope of $\S 5$ is to establish

Theorem 1. Let $M$ be a compact strictly pseudoconvex $C R$ manifold. If $\delta>0$ and $\theta, \hat{\theta} \in \mathcal{P}_{+}$are two contact forms on $M$ such that $d(\theta, \hat{\theta})<\delta$ then $e^{-\delta} \lambda_{k}(\theta) \leq \lambda_{k}(\hat{\theta}) \leq e^{\delta} \lambda_{k}(\theta)$ for any $k \geq 0$.

Proof. For any $x \in M$

$$
\delta>\inf \left\{\epsilon>0: e^{-\epsilon} G_{\theta, x}<G_{\hat{\theta}, x}<e^{\epsilon} G_{\theta, x}\right\}
$$

i.e. there is $0<\epsilon<\delta$ such that $G_{\hat{\theta}, x}-e^{-\epsilon} G_{\theta, x} \in P(H)_{x}$ and $e^{\epsilon} G_{\theta, x}-G_{\hat{\theta}, x} \in$ $P(H)_{x}$. There is a unique $u \in C^{\infty}(M, \mathbb{R})$ such that $\hat{\theta}=e^{u} \theta$. Consequently

$$
\hat{\theta} \wedge(d \hat{\theta})^{n}=e^{(n+1) u} \theta \wedge(d \theta)^{n} .
$$

On the other hand $e^{-\delta} G_{\theta, x}(v, v)<G_{\hat{\theta}, x}(v, v)<e^{\delta} G_{\theta, x}(v, v)$ for any $v \in$ $H(M)_{x} \backslash\{0\}$ implies $|u|<\delta$. Then for every $f \in C^{\infty}(M)$ (by (11))

$$
e^{-(n+1) \delta} \int_{M} f^{2} \Psi_{\theta} \leq \int_{M} f^{2} \Psi_{\hat{\theta}} \leq e^{(n+1) \delta} \int_{M} f^{2} \Psi_{\theta} .
$$

Moreover

$$
\hat{\nabla}^{H} f=e^{-u} \nabla^{H} f
$$

where $\hat{\nabla}^{H} f$ is the horizontal gradient of $f$ with respect to $\hat{\theta}$. Thus (by (13)) $\left\|\hat{\nabla}^{H} f\right\|_{\hat{\theta}}^{2}=e^{-u}\left\|\nabla^{H} f\right\|_{\theta}^{2}<e^{\delta}\left\|\nabla^{H} f\right\|_{\theta}^{2}$ so that (by (11))

$$
\begin{gathered}
e^{-(n+2) \delta} \int_{M}\left\|\nabla^{H} f\right\|_{\theta}^{2} \Psi_{\theta} \leq \int_{M}\left\|\hat{\nabla}^{H} f\right\|_{\hat{\theta}}^{2} \Psi_{\hat{\theta}} \leq \\
\leq e^{(n+2) \delta} \int_{M}\left\|\nabla^{H} f\right\|_{\theta}^{2} \Psi_{\theta} .
\end{gathered}
$$

Finally (by (12)-(13))

$$
e^{-\delta} \frac{\left\|\nabla^{H} f\right\|_{L^{2}}^{2}}{\|f\|_{L^{2}}^{2}} \leq \frac{\int_{M}\left\|\hat{\nabla}^{h} f\right\|_{\hat{\theta}}^{2} \Psi_{\hat{\theta}}}{\int_{M} f^{2} \Psi_{\hat{\theta}}} \leq e^{\delta} \frac{\left\|\nabla^{H} f\right\|_{L^{2}}^{2}}{\|f\|_{L^{2}}^{2}}
$$

so that (by the Max-Mini principle)

$$
e^{-\delta} \lambda_{k}(\theta) \leq \lambda_{k}(\hat{\theta}) \leq e^{\delta} \lambda_{k}(\theta) .
$$

Theorem 1 is proved. Corollary 1 follows from (15). 


\section{SPECTRA OF $\Delta_{b}$ AND $\square$}

Let $F_{\theta}$ be the Fefferman metric of $(M, \theta)$ and $\square$ the corresponding wave operator (the Laplace-Beltrami operator of $\left(C(M), F_{\theta}\right)$ ). We set $\mathfrak{M}=C(M)$ for simplicity. Let $g$ be a fixed Riemannian metric on $\mathfrak{M}$. The space $S(\mathfrak{M})$ of all symmetric tensor fields may be identified with the space of all fields of endomorphisms of $T(\mathfrak{M})$ which are symmetric with respect to $g$ i.e. for each $h \in S(\mathfrak{M})$ let $\tilde{h} \in C^{\infty}(\operatorname{End}(T(\mathfrak{M})))$ be given by

$$
g(\tilde{h} X, Y)=h(X, Y), \quad X, Y \in \mathfrak{X}(\mathfrak{M}) .
$$

From now on we assume that $M$ is compact. Then $\mathfrak{M}$ is compact as well (as $\mathfrak{M}$ is the total space of a principal bundle with compact base and compact fibres) and we endow $S(\mathfrak{M})$ with the distance function

$$
d_{g}^{\infty}\left(h_{1}, h_{2}\right)=\sup _{z \in \mathfrak{M}}\left[\operatorname{trace}\left(\varphi_{z}^{2}\right)\right]^{1 / 2}, \quad h_{1}, h_{2} \in S(\mathfrak{M}),
$$

where $\varphi=\tilde{h}_{1}-\tilde{h}_{2}$ and $\varphi_{z}^{2}=\varphi_{z} \circ \varphi_{z}$. The set Lor $(\mathfrak{M})$ of all Lorentz metrics on $\mathfrak{M}$ is an open set of $\left(S(\mathfrak{M}), d_{g}^{\infty}\right)$ and for any pair $g_{1}, g_{2}$ of Riemannian metrics on $\mathfrak{M}$ the distance functions $d_{g_{1}}$ and $d_{g_{2}}$ are uniformly equivalent (cf. e.g. [10], p. 49). We shall use the topology induced by $d_{g}^{\infty}$ on $\operatorname{Lor}(\mathfrak{M})$ (and therefore on $C \subset \operatorname{Lor}(\mathfrak{M})$ ). By a result of J.M. Lee, [8], the sublaplacian $\Delta_{b}$ of $(M, \theta)$ is the pushforward of the wave operator i.e. $\pi_{*} \square=\Delta_{b}$. In particular $\sigma\left(\Delta_{b}\right) \subset \sigma(\square)$. Thus each $\lambda_{k}: \mathcal{P}_{+} \rightarrow \mathbb{R}$ may be thought of as a function $\lambda_{k}^{\uparrow}: C \rightarrow \mathbb{R}$ such that $\lambda_{k}^{\uparrow} \circ F=\lambda_{k}$ for every $k \geq 0$, where $F: \mathcal{P}_{+} \rightarrow C$ is the map given by $F(\theta)=F_{\theta}$ for every $\theta \in \mathcal{P}_{+}$. As another consequence of Theorem 1 we establish

Corollary 2. Let $M$ be a compact strictly pseudoconvex $C R$ manifold and let $g$ be an arbitrary Riemannian metric on $\mathfrak{M}=C(M)$. Let $\theta_{0} \in \mathcal{P}_{+}$be a fixed contact form and $\mathcal{P}_{++}=\left\{e^{u} \theta_{0}: u \in C^{\infty}(M, \mathbb{R}), u>0\right\}$. If $C_{+}=\left\{F_{\theta}\right.$ : $\left.\theta \in \mathcal{P}_{++}\right\}$then for every $k \in \mathbb{N} \cup\{0\}$ the function $\lambda_{k}^{\uparrow}: C_{+} \rightarrow \mathbb{R}$ is continuous relative to the $d_{g}^{\infty}$-topology.

Proof. Let $\theta_{i} \in \mathcal{P}_{+}, i \in\{1,2\}$, and let us set $\varphi=\tilde{F}_{\theta_{1}}-\tilde{F}_{\theta_{2}}$. Let $\left\{E_{p}: 1 \leq\right.$ $p \leq 2 n+2\}$ be a local $g$-orthonormal frame on $T(\mathfrak{M})$, defined on the open set $\mathcal{U} \subset \mathfrak{M}$. Then

$$
\operatorname{trace}\left(\varphi^{2}\right)=\sum_{p=1}^{2 n+2} g\left(\varphi^{2} E_{p}, E_{p}\right)=\sum_{p}\left\{F_{\theta_{1}}\left(\varphi E_{p}, E_{p}\right)-F_{\theta_{2}}\left(\varphi E_{p}, E_{p}\right)\right\}
$$

on $\mathcal{U}$. On the other hand if $\varphi E_{p}=\varphi_{p}^{q} E_{q}$ then $\varphi_{p}^{q}=F\left(\theta_{1}\right)\left(E_{p}, E_{q}\right)-$ $F\left(\theta_{2}\right)\left(E_{p}, E_{q}\right)$ hence

$$
\operatorname{trace}\left(\varphi^{2}\right)=\left(e^{u_{1} \circ \pi}-e^{u_{2} \circ \pi}\right)^{2}\left\|F_{\theta_{0}}\right\|_{g}^{2}
$$


where $u_{i} \in C^{\infty}(M, \mathbb{R})$ is given by $\theta_{i}=e^{u_{i}} \theta_{0}$ and $\left\|F_{\theta_{0}}\right\|_{g}$ is the norm of $F_{\theta_{0}}$ as a $(0,2)$-tensor field on $\mathfrak{M}$ with respect to $g$. Then (by (16))

$$
d_{g}^{\infty}\left(F_{\theta_{1}}, F_{\theta_{2}}\right)=\sup _{\mathfrak{M}}\left|e^{u_{1} \circ \pi}-e^{u_{2} \circ \pi}\right||| F_{\theta_{0}} \|_{g} .
$$

As $\mathfrak{M}$ is compact $a=\inf _{z \in \mathfrak{M}}\left\|F_{\theta_{0}}\right\|_{g, z}>0$. Indeed (by compactness) $a=$ $\left\|F_{\theta_{0}}\right\|_{g, z_{0}}$ for some $z_{0} \in \mathfrak{M}$. If $a=0$ then $F_{\theta_{0}, z_{0}}=0$, a contradiction (as $F_{\theta_{0}}$ is Lorentzian, and hence nondegenerate). Let $\epsilon>0$ such that $d_{g}^{\infty}\left(F_{\theta_{1}}, F_{\theta_{2}}\right)<$ $\epsilon$. Then $\left|e^{u_{1}}-e^{u_{2}}\right|<\epsilon / a$ everywhere on $M$. As both $u_{1}>0$ and $u_{2}>0$ it follows that $\left|u_{1}-u_{2}\right|<\log (1+\epsilon / a)$. Indeed $e^{u_{1}}-e^{u_{2}}<\epsilon / a$ is equivalent to $e^{u_{1}-u_{2}}<1+(\epsilon / a) e^{-u_{2}}$ hence (as $\left.u_{2}>0\right)$

$$
u_{1}-u_{2}<\log \left[1+(\epsilon / a) e^{-u_{2}}\right]<\log (1+\epsilon / a) .
$$

Therefore

$$
(1+\epsilon / a)^{-1} G_{\theta_{1}, x}(v, v)<G_{\theta_{2}, x}(v, v)<(1+\epsilon / a) G_{\theta_{1}, x}(v, v)
$$

for any $v \in H(M)_{x} \backslash\{0\}$ and any $x \in M$. Consequently $\rho_{H}^{\prime \prime}\left(G_{\theta_{1}}, G_{\theta_{2}}\right)<$ $\log (1+\epsilon / a)$. The arguments in $\S 5$ then yield

$$
(1+\epsilon / a)^{-1} \lambda_{k}^{\uparrow}\left(F_{\theta_{1}}\right) \leq \lambda_{k}^{\uparrow}\left(F_{\theta_{2}}\right) \leq(1+\epsilon / a) \lambda_{k}^{\uparrow}\left(F_{\theta_{1}}\right)
$$

and Corollary 2 follows. The problem of the behavior of $\lambda_{k}^{\uparrow}: C \rightarrow \mathbb{R}$ is open. So does the more general problem of the behavior of the spectrum of the wave operator on $\mathfrak{M}$ with respect to a change of $F \in \operatorname{Lor}(\mathfrak{M})$. Further work (cf. [1]) on the behavior of $\sigma\left(\Delta_{b}\right)$ under analytic 1-parameter deformations $\{\theta(t)\}_{t \in \mathbb{R}}$ of a given contact form $\theta_{0} \in \mathcal{P}_{+}$builds on the Riemannian counterpart in [6] and the functional analysis results in [7].

\section{REFERENCES}

[1] A. Aribi \& S. Dragomir \& A. El Soufi, Sublaplacian eigenvalue functionals and contact structure deformations on compact CR manifolds, in preparation.

[2] S. Bando \& H. Urakawa, Generic properties of the eigenvalues of the Laplacian for compact Riemannian manifolds, Tohoku Math. J., 35(1983), 155-172.

[3] E. Barletta \& S. Dragomir, Sublaplacians on CR manifolds, Bull. Math. Soc. Sci. Math. Roumanie, Tome 52(100) No. 1, 2009, 3-32.

[4] J.M. Bony, Principe du maximum, inégalité de Harnak et unicité du problème de Cauchy pour les opérateurs elliptiques dégénéré, Ann. Inst. Fourier, Grenoble, (1)19(1969), 277-304.

[5] S. Dragomir \& G. Tomassini, Differential Geometry and Analysis on CR manifolds, Progress in Mathematics, Vol. 246, Birkhäuser, Boston-Basel-Berlin, 2006.

[6] A. El Soufi \& S. Ilias, Laplacian eigenvalue functionals and metric deformations on compact manifolds, J. Geom. Phys., 58(2008), 89-104.

[7] A. Kriegl \& P. Michor, Differentiable perturbation of unbounded operators, Math. Ann., (1)327(2003), 191-201.

[8] J.M. Lee, The Fefferman metric and pseudohermitian invariants, Trans. A.M.S., (1)296(1986), 411-429. 
[9] A. Menikoff \& J. Sjöstrand, On the eigenvalues of a class of hypoelliptic operators, Math. Ann., 235(1978), 55-85.

[10] P. Mounoud, Some topological properties of the space of Lorentz metrics, Differential Geometry and its Applications, 15(2001), 47-57.

[11] W. Rudin, Functional analysis, International Series in Pure and Applied Mathematics, McGraw-Hill, Inc., New York-London-Paris, 1991.

[12] J. Sjöstrand, On the eigenvalues of a class of hypoelliptic operators. IV, Annales de l'institut Fourier, tome 30, n. 2 (1980), p. 109-169.

[13] H. Urakawa, How do eigenvalues of Laplacian depend upon deformations of Riemannian metrics?, Spectra of Riemannian manifolds, Kaigai Publications, Tokyo, 1983, 129-137. 